\title{
Summary of the Heavy Flavor Working Group
}

\author{
Michael Klasen ${ }^{1}$, Benno List ${ }^{2}$, Stephanie Hansmann-Menzemer ${ }^{3}$, and Rainer Mankel ${ }^{4}$ \\ 1- Université Grenoble I - Laboratoire de Physique Subatomique et de Cosmologie \\ 53 Avenue des Martyrs - 38026 Grenoble - France \\ 2- Universität Hamburg - Institut für Experimentalphysik \\ Luruper Chaussee 149 - 22761 Hamburg - Germany \\ 3- Universität Heidelberg - Physikalisches Institut \\ Philosophenweg 12 - 69120 Heidelberg - Germany \\ 4- Deutsches Elektronen-Synchrotron DESY - F1 \\ Notkestr. 85 - 22603 Hamburg - Hamburg
}

\begin{abstract}
During the last year many important results have been achieved in heavy flavour physics: New measurements of charm and beauty production have been performed at HERA and the Tevatron. A wealth of new spectroscopy data with several new, unexpected states in the charmonium and the $D_{\mathrm{s}}$ systems has been collected and $b \rightarrow d \gamma$ transitions have been established. The oscillation frequency in the $B_{\mathrm{s}} \bar{B}_{\mathrm{s}}$ is now measured, and mixing in the $D^{0} \bar{D}^{0}$ system has been observed. Theoretical progress in the areas of open heavy flavour production, quarkonium production and decays, and multiquark spectroscopy has been presented at this workshop.
\end{abstract}

\section{Experimental Summary}

In this section we summarize the experimental results from the heavy flavour working group [1]. The presentations covered a wide range of topics, from charm, beauty and charmonium production in $e p$ and $p \bar{p}$ collisions, heavy ion results on charm suppression, spectroscopy and rare decays, over oscillations in the $B_{\mathrm{s}} \bar{B}_{\mathrm{s}}$ and $D^{0} \bar{D}^{0}$ systems to the outlook for heavy flavour physics at future experiments at the LHC and the ILC.

\subsection{Charm and Beauty Production}

\subsubsection{Charm Production}

Charm Production in ep collisions has been studied extensively over the last years at HERA, and a wealth of data exist in photoproduction (where the exchanged photon is quasi-real, with a virtuality $Q^{2} \approx 0$ ) and deep-inelastic scattering (DIS). The HERA collider experiments, H1 and ZEUS, have presented new preliminary results on charm production from the HERA-II running period $[2,3,4]$. The ZEUS Collaboration reported on two charm measurements in DIS with HERA-II data (Fig. 1): One analysis [2] uses $D^{*}$ mesons to identify charm production, and utilizes $162 \mathrm{pb}^{-1}$ of new data to achieve improved statistical accuracy compared to previous analyses. The second analysis [3] uses $D^{ \pm}$mesons instead and is one of the first measurements to utilize the new silicon strip Micro Vertex Detector (MVD) of ZEUS. Based on $135 \mathrm{pb}^{-1}$, this analysis achieves similar accuracies as previous ZEUS measurements of the inclusive charm cross section in DIS.

The ZEUS collaboration has recently also finalized two analyses of charm production from older HERA-I data [2]. One analysis [5] covers the region of very low momentum transfer $\left(0.05<Q^{2}<0.7 \mathrm{GeV}^{2}\right)$ at the transition between photoproduction and DIS. The data provide a good test of perturbative QCD calculations, which are available in NLO, and are 
well described by massive calculations in the fixed flavour number scheme, as implemented in the HVQDIS program [6]. In the second analysis [7], the pseudoscalar states $D^{0}, D^{+}$, and $D_{\mathrm{s}}^{+}$are reconstructed, rather than the vector state $D^{*+}$, which allows a measurement of the fragmentation fractions, which turn out to be compatible with those measured in $e^{+} e^{-}$ annihilation and in photoproduction $[8,9]$. The inclusive charm cross section derived from this measurement is consistent with previous results. Also a new, preliminary measurement of charm fragmentation was presented by the ZEUS collaboration [8], which shows broad agreement with other measurements from $\mathrm{H} 1$ [4], and also with measurements from $e^{+} e^{-}$ experiments.

Another new measurement of $D^{*}$ production in DIS, based on $222 \mathrm{pb}^{-1}$ of HERA-II data, was presented by the $\mathrm{H} 1 \mathrm{col}-$ laboration [4]. Here, differential and double differential visible cross sections for $D^{*}$ production were measured and compared to the QCD calculations. Overall, these quantities are well described by the NLO predictions of HVQDIS; in fact, the data uncertainties are in many cases smaller than the theory uncertainties from the variation of the charm mass and the variation of the renormalization and factorization scales. However, in some quantities, most notably the $\eta\left(D^{*}\right)$ distribution, even in spite of the relatively large theory uncertainties the NLO predictions deviate significantly from the trend observed in data.

This confirms, with higher statistics, the observation made in a recently published analysis from the H1 collaboration [10, 11]. However, that analysis goes one step further and investigates $D^{*}$ production in DIS in conjunction with jets. As heavy flavour production is dominated by boson gluon fusion, a two-jet structure is expected for most of the events. In the DIS analysis, one jet in addition to the $D^{*}$ meson is required.
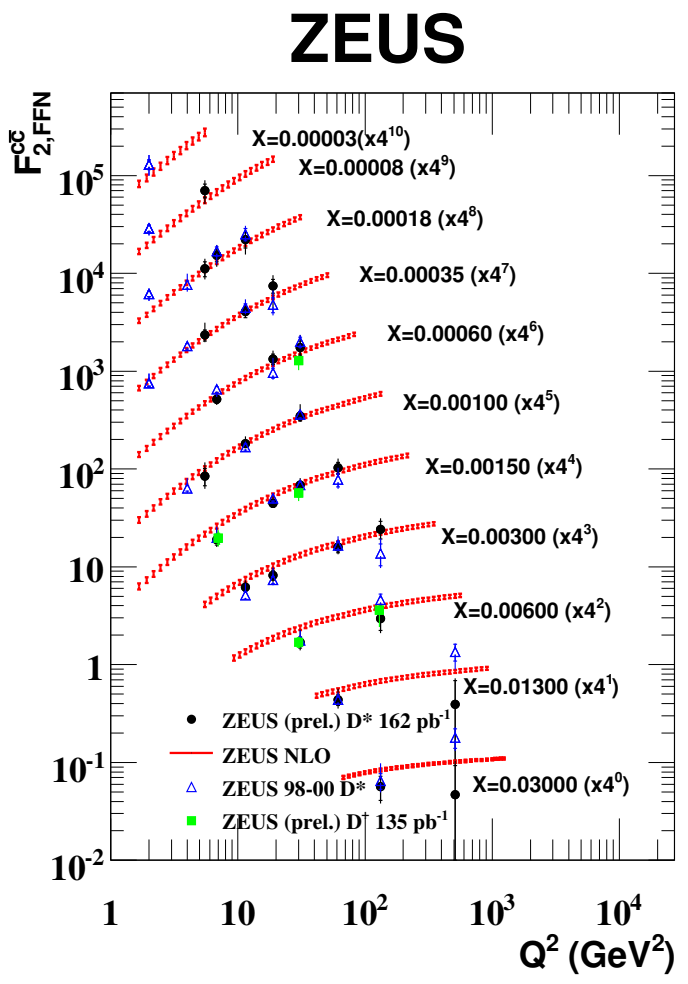

Figure 1: Compilation of $F_{2}^{c}$ measurements from ZEUS.

Again, HVQDIS describes the data satisfac-

torily, with the notable exception of the azimuthal angle difference $\Delta \phi$ between the $D^{*}$ and the jet, a quantity which would be almost exactly equal to $180^{\circ}$ in leading order, where the two charm jets must be back-to-back; hard gluon emission, which enters only in NLO, leads to an enhancement at lower values of $\Delta \phi$, which is underestimated by HVQDIS, whereas Monte Carlo models such as CASCADE [12], which include parton showers as approximation for higher-order gluon radiation, work significantly better. In another recent publication $[10,13]$, the H1 collaboration has investigated charm photoproduction with two jets, with very similar findings.

The results show that charm measurements have now reached a level of accuracy where 
more precise QCD predictions would be desirable. On one hand a full NLO Monte Carlo with matched parton showers is needed, as opposed to HVQDIS, which produces only parton fourvectors and has been augmented with relatively simple independent fragmentation routines to make the extraction of visible cross sections possible. A second way to reduce theory uncertainties would be to reduce the variation of input parameters such as the charm mass or fragmentation parameters by detailed comparisons between MC predictions and data of (double) differential visible cross sections in terms of relevant quantities such as $p_{\mathrm{t}}\left(D^{*}\right)$ and $\eta\left(D^{*}\right)$. On the other hand, NNLO calculations would provide the most reliable way to reduce theory uncertainties, in particular for inclusive quantities such as $F_{2}^{c \bar{c}}$.

\subsubsection{Beauty Production}

Beauty production at HERA and the Tevatron has generated a lot of interest in recent years, not least because at both colliders inclusive cross sections for beauty production were observed that are considerably higher than the expectation from perturbative QCD calculations in next-to-leading order.

Achim Geiser gave an overview over the existing experimental results from HERA, with additional glimpses at Tevatron and UA1 data [14]. He discussed in detail the issue of scale dependence of QCD calculations, where he presented a survey of QCD calculations in different orders of $\alpha_{\mathrm{s}}$ for a number of processes and argued that a good choice for the factorisation and renormalisation scales $\mu_{\mathrm{r}, \mathrm{f}}$ would be a scale at which higher order calculations give a result that coincides with the calculations at lower order. He observed that this is achieved in most of the cases at a scale between $1 / 4 \mu_{0}$ and $1 \mu_{0}$, where $\mu_{0}$ is the "natural" choice of scale such as $E_{\mathrm{T}}^{2}, Q^{2}+m_{\mathrm{Q}}^{2}$, or $p_{\mathrm{t}}^{2}+m_{\mathrm{Q}}^{2}$. Based on this observation he argued that the factorisation and renormalisation scales should be varied in the range $1 / 4 \mu_{0}<\mu_{\mathrm{r}, \mathrm{f}}<1 \mu_{0}$, with a choice of $\mu_{\mathrm{r}, \mathrm{f}}=1 / 2 \mu_{0}$ for the central value, rather than using a variation of $1 / 2 \mu_{0}<\mu_{\mathrm{r}, \mathrm{f}}<2 \mu_{0}$, as is customary in most HERA publications. He also pointed out that this prescription has already been adopted silently by the Tevatron experiments in recent preliminary results. This proposal was received well by the audience, as the discussion after the presentation showed.

New, preliminary results on beauty production were presented by the D0 and CDF collaborations [15]. D0 has performed a new measurement of muon tagged jet cross sections based on $300 \mathrm{pb}^{-1}$ of data that extends up to $p_{\mathrm{t}}=420 \mathrm{GeV}$. Again, the data lie above the theory prediction. In a new measurement of inclusive B cross sections, the CDF collaboration utilizes the decays $B \rightarrow \ell D^{0}, D^{*+} X$ in addition to the previously used channel and $B \rightarrow$ $J / \psi X$, with consistent results. These measurements are also consistent with a fixed order calculation at next to leading log.

The first, preliminary HERA-II result on beauty production, based on $39 \mathrm{pb}^{-1}$ of data, was presented by the ZEUS collaboration [16]. The results are somewhat higher, but still consistent with previous measurements from $\mathrm{H} 1$ [17].

\subsection{Charmonium Production}

The inclusive production of charmonium and bottomium states in $e p$ and $p \bar{p}$ collisions remains an intersting testing ground for perturbative QCD, as was shown in a review by Katja Krüger [18]. The working horse for these investigations are $J / \psi$ mesons, where the largest data sets are available. While leading order calculations in the colour singlet model [19] fail 
to describe the production rates of $J / \psi$ mesons at the Tevatron [20], NRQCD models [21] models have been successfully applied there. In these models the production of $c \bar{c}$ states with different colour and spin/parity configuration is calculated perturbatively, and the transition to bound states is described by non-perturbative long distance matrix elements (LDME), which have to be determined from data. This allows the production of $J / \psi$ mesons by gluon splitting into a $c \bar{c}$ pair, followed by a transition to a $J / \psi$ meson, which is predicted to be transversely polarized. The observation of such a polarization is therefore considered the "smoking gun" for (large) colour octet (CO) contributions.

However, new data from CDF that were presented at this conference [15] show a clear evidence for longitudinal $J / \psi$ polarization, which is in contrast to NRQCD expectations. New data on $\psi^{\prime}$ polarisation are still not precise enough for firm conclusions.

A second test of NRQCD predictions is the measurement of the production rates of $\chi_{\mathrm{c} 2}$ versus $\chi_{\mathrm{c} 1}$ states, which in NRQCD models is expected to follow the spin counting prediction of $5 / 3$. New measurements from CDF $[22,15]$ give a significantly smaller result for the ratio of prompt $\chi_{\mathrm{c}}$ production: $\sigma\left(\chi_{\mathrm{c} 2}\right) / \sigma\left(\chi_{\mathrm{c} 1}\right)=0.70 \pm 0.04($ stat. $) \pm 0.04($ syst. $) \pm 0.06(B F)$.

After the initial success of the NRQCD model indications for the necessity of large CO contributions where also searched for in inelastic $J / \psi$ production at HERA. The H1 collaboration has presented new, preliminary data from HERA-II on inelastic $J / \psi$ production in deep inelastic $e p$ scattering [23]. Production rates were measured double differentially in the transverse momentum $p_{\mathrm{t}}$ and the momentum fraction $z$ of the $J / \psi$ meson, and compared to Monte Carlo predictions by programs which implement the colour singlet model in leading order. These models describe the shape of the measurements quite well, whereas the $\mathrm{CO}$ contributions in NRQCD models tend to have different $p_{\mathrm{t}}$ and $z$ shapes than the CS contributions. Therefore, the new $\mathrm{H} 1$ data do not show any clear need for large $\mathrm{CO}$ contributions to inelastic $J / \psi$ production. In the discussion of the results the need was stressed for NLO calculations of this process, in the CS as well as in NRQCD models. NLO calculations are currently only available in the CS model for photoproduction of $J / \psi$ mesons [24], where they describe the data from HERA [25] quite well.

A different issue in charmonium production is adressed by the new data from HERA-B [26]: HERA-B has collected large samples of $J / \psi$ and $\psi^{\prime}$ mesons decaying to $\mu^{+} \mu^{-}$and $e^{+} e^{-}$ pairs, which made it possible to investigate the dependence of charmonium production on the atomic weight $A$ in a new range of Feynman- $x_{\mathrm{F}}$, extending the range covered by the experiments down to $x_{\mathrm{F}}=-0.35$, which is a region where theoretical models make widely different predictions.

\subsection{Heavy Ion Results}

The new results from heavy ion experiments at RHIC have been reviewed in this conference by William Zajc [27]. Therefore we only briefly highlight some of the new results presented in the heavy flavour session.

New results on heavy quark production in $\mathrm{Au}+\mathrm{Au}$ collisions were presented by the PHENIX [28] and the STAR [29] collaborations. Due to their larger mass and the dead cone effect, charm quarks are expected to lose energy at a smaller rate than light quarks. The observation of supression of electrons from charm decays in central $\mathrm{Au}+\mathrm{Au}$ collisions $[28,30,29,31]$ therefore comes as a surprise. It appears that charm quarks participate in the flow of the opaque hadronic medium just as much as light quarks, which means that they thermalize more quickly than expected in many theoretical models. 
The PHENIX collaboration has also presented new measurements of $J / \psi$ suppression in $\mathrm{Au}+\mathrm{Au}$ collisions $[32,33]$. These measurements, which are performed in two rapidity ranges $|y|<0.35$ and $1.2<y<2.2$, show a significant suppression of $J / \psi$ production in $\mathrm{Au}+\mathrm{Au}$ collisions, which is stronger at large rapidities than at central rapidity values. The explanation of this rapidity dependence is a real challenge to theoretical models.

\subsection{Spectroscopy and Rare Decays}

The large data sets from the B factory experiments BaBar and Belle, from CLEO-c and from the Tevatron experiments CDF and D0 have lead to a renewed interest in the spectroscopy of charm and bottom hadrons with beautyful results, and have opened the possibility to identify extremely rare decays.

\subsubsection{The Charmonium System}

In the charmonium system, finally all expected charmonium states below the D-meson threshold have now been firmly identified. One of last two missing states, the $\eta_{\mathrm{c}}^{\prime}$, has now seen by BaBar, CLEO-c, and Belle [34, 35], at an average mass of $m\left(\eta_{\mathrm{c}}^{\prime}\right)=3638 \pm 4 \mathrm{MeV}$. This allows a comparison of the hyper fine splitting of the $1 S$ and $2 S$ states in the charmonium system, which are $\Delta m_{\mathrm{hf}}(1 S)=117 \pm 1 \mathrm{MeV}$ and $\Delta m_{\mathrm{hf}}(2 S)=48 \pm 4 \mathrm{MeV}$; the large difference of these values poses a challenge to theory [34].

The long elusive $h_{\mathrm{c}}$ state has observed by CLEO-c $[34,36]$ in the decay chain $\psi(2 S) \rightarrow$ $\pi^{0} h_{\mathrm{c}}, h_{\mathrm{c}} \rightarrow \gamma \eta_{\mathrm{c}}$, with a mass of $m\left(h_{\mathrm{c}}\right)=3524.4 \pm 0.6 \pm 0.4 \mathrm{MeV}$, which corresponds to a hyper fine splitting between the $h_{\mathrm{c}}$ and the center of gravity of the $\chi_{\mathrm{c} 0,1,2}$ states of $m_{\mathrm{hf}}(1 P)=$ $+1.0 \pm 0.6 \pm 0.4 \mathrm{MeV}$, consistent with the expected value of zero. Meanwhile, CLEO-c has increased its $\psi(2 S)$ sample eightfold, which yield the promise of further, improved results.

In addition to these expected charmonium states, recent years have seen the discovery of several unexpected charmonium-like resonances:

New results were obtained on the $X(3872)$, which is already considered firmly established by the PDG [37], and on the $Y(3940)$ and $Y(4260)$. Results were also presented on two additional states, the $X(3940)$ and the $Z(3930)$.

For the $X(3872)$, one explanation that has been put forward is the interpretation as a $D^{0} \overline{D^{0} *}$ molecule. CLEO has performed a new measurement of the $D^{0}$ mass [34]: $m\left(D^{0}\right)=$ $1864.847 \pm 0.150$ (stat.) \pm 0.095 (syst.) MeV [38]. The total uncertainty of this measurement of $0.178 \mathrm{MeV}$ is a factor of 2.2 better than the uncertainty of $0.4 \mathrm{MeV}$ of the 2006 world average [37]. Combinig this result with the PDG06 value of the $X(3872)$ mass of $3871.2 \pm 0.5 \mathrm{MeV}$ results in a very small binding energy of $E_{\mathrm{b}}=0.6 \pm 0.6 \mathrm{MeV}$ for a $D^{0} \overline{D^{0}}$ molecule [34]. Meanwhile, Belle and BaBar have also found indications for the $X(3872)$ in the decays $B \rightarrow \bar{D}^{0} D^{0} \pi^{0} K$ (Belle) and $B \rightarrow \bar{D}^{0} D^{0 *} K$ (BaBar) [39]. In these channels, the observed mass values for the $X(3872)$ of $m=3875.4 \pm 0.7_{-2.0}^{+1.2} \mathrm{MeV}$ (Belle) [40] and $m=3875.6 \pm$ $0.7_{-1.5}^{+1.4} \mathrm{MeV}$ (BaBar) are $2.5 \sigma$ higher than the current world average. Belle also concludes that the quantum numbers $J^{P C}=1^{++}$are favoured if the observed enhancement is indeed the $X(3872)$. All in all, the interpretation of this state remains unclear.

New data were also presented on the $Y(4260)$ [39], which was first observed in initial state radiation events at BaBar. BaBar sets a limit [41] of $B R(Y(4260) \rightarrow D \bar{D}) / B R(Y(4260) \rightarrow$ $\left.J / \psi \pi^{+} \pi^{-}\right)<7.6$, which is a further indication that the $Y(4260)$ is not a conventional charmonium state. CLEO has also confirmed the $Y(4260)[34,42]$, and finds [43] its mass to be 
$m=4284_{-16}^{+17}$ (stat) \pm 4 (syst.) MeV, in poor agreement with the original BaBar measurement [44] of $m=4259 \pm 8_{-6}^{+2} \mathrm{MeV}$. The latest Belle [39, 45] result $m=4295 \pm 10_{-3}^{+10} \mathrm{MeV}$ is consistent with the CLEO measurement.

The discovery of new, unexpected charmonium-like states has also triggered new investigations of $R=\sigma\left(e^{+} e^{-} \rightarrow\right.$ hadrons $) / \sigma\left(e^{+} e^{-} \rightarrow \mu^{+} \mu^{-}\right)$at Belle and CLEO [46, 42, 43, 45]. These scans show a marked dip around the $Y(4260)$. Belle, BaBar and CLEO have also looked $[46,41,47,48]$ into the more exclusive channels $e^{+} e^{-} \rightarrow D^{(*)} D^{(*)}$. A weak signal for the $Y(4260)$ is seen in the $D \bar{D}^{*}$ channel, no signal in the $D D$ channel, and a dip, similar to the one observed in the inclusive $R$ measurement, is observed in the $D^{*} D^{*}$ channel around the $Y(4260)$ resonance.

Meanwhile, BaBar observes yet another state $[39,49]$ in initial state production of $e^{+} e^{-} \rightarrow \psi(2 S) \pi^{+} \pi^{-}$at a mass of $m=4324 \pm 24 \mathrm{MeV}$ and a width of $\Gamma=172 \pm 33 \mathrm{MeV}$, which is incompatible with the $Y(4260)$ or other known states such as the $\psi(4415)$.

Three more states observed by Belle [39, 50], termed $X(3940), Y(3940)$, and $Z(3930)$, may have an interpretation as conventional charmonium states, namely the $\eta_{\mathrm{c}}(3 S)\left[3{ }^{1} S_{0}\right]$, the $\chi_{\mathrm{c} 1}^{\prime}\left[2^{3} P_{1}\right]$ and the $\chi_{\mathrm{c} 2}^{\prime}\left[2^{3} P_{2}\right]$.

\subsubsection{Charmed, Strange Mesons}

In the sector of charmed, strange mesons new measurements were presented by BaBar and Belle [39]. In addition to new measurements of the properties of the $D_{\mathrm{s} 0}^{*}(2317)$ and $D_{\mathrm{s} 1}(2460)$, another new state, termed $D_{\mathrm{sJ}}^{*}(2860)$, has been identified by BaBar [51] with a mass $m=2856.6 \pm 1.5 \pm 5.0 \mathrm{MeV}$ and a spin parity assignment $J^{P}=0^{+}, 1^{-}, 2^{+}, \ldots$. In addition, a hint for another state with $m=2688 \pm 4 \pm 3 \mathrm{MeV}$ has also been observed. Furthermore, Belle, in a Dalitz analysis of the decay $B^{+} \rightarrow \bar{D}^{0} D^{0} K^{+}$, sees indications for a state $D_{\text {sJ }}(2700)$ with $m=2715 \pm 11_{-14}^{+11} \mathrm{MeV}$, with quantum numbers $J^{P}=1^{-}$favoured. The theoretical interpretation of these states is not yet clear. While the $D_{\mathrm{s} 0}^{*}(2317)$ and $D_{\mathrm{s} 1}(2460)$ states can be explained as $0^{+}$and $1^{+} \mathrm{P}$-wave $c \bar{s}$ states, their masses are substantially lower than expected from potential models. The interpretation of the other states is still less clear.

\subsubsection{Charmed Baryons}

Coming to charmed baryons, BaBar and Belle have reported discoveries of several new states [46]. Both observe the new state $\Lambda_{\mathrm{c}}(2940)^{+}$in the channels $D^{0} p$ (BaBar) and $\Lambda_{\mathrm{c}}^{+} \pi^{+} \pi^{-}$ (Belle) [52]. The Belle discovery of the new charmed, strange baryons $\Xi_{\mathrm{c}}(2980)^{+}$and $\Xi_{\mathrm{c}}(3077)^{+}$has been confirmed by BaBar [53], and Belle sees also some evidence for the isospin partners $\Xi_{\mathrm{c}}(2980)^{0}$ and $\Xi_{\mathrm{c}}(3077)^{0}$. BaBar has also reported the first observation of the $\Omega_{\mathrm{c}}^{*}$, an excited css state [54].

\subsubsection{Bottom Mesons}

In the B meson sector, the progress comes from the Tevatron experiments CDF and D0 [55]. The $B_{\mathrm{c}}^{+}$has already been observed by both experiments, now CDF reports the first direct observation of the $B_{\mathrm{c}}^{+}$in the exclusive decay channel $B_{\mathrm{c}}^{+} \rightarrow J / \psi \pi^{+}$, which allows a very precise mass measurement with the preliminary result of $m\left(B_{\mathrm{c}}^{+}\right)=6276.5 \pm 4.0 \pm 2.7 \mathrm{MeV}$, while the uncertainty on the old world average was $400 \mathrm{MeV}$. The $\eta_{\mathrm{b}}$ is last spin singlet ground state in the bottomium sector that has not been unambiguously discovered yet. CDF 
has performed a new search for the decay $\eta_{\mathrm{b}} \rightarrow J / \psi J / \psi$, without indications for a signal. Both, D0 and CDF, report first direct observations on orbitally excited B mesons with $L=1$ by looking for decays $B^{* *} \rightarrow B^{(*)+} \pi^{-}$. Both see clear evidence for $B_{1}$ and $B_{2}$ states. With a similar analysis of the channels $B_{\mathrm{s}}^{* *} \rightarrow B^{(*)}+K^{-}$both experiments observe also the $B_{\mathrm{s} 2}^{*}$ state, in addition CDF reports evidence for the $B_{\mathrm{s} 1}$ state.

\subsubsection{Bottom Baryons}

In the bottom baryon sector, where up to now the $\Lambda_{\mathrm{b}}^{0}$ is the only well established particle, CDF performed a blind analysis of the decay channel $\Lambda_{\mathrm{b}}^{0} \pi^{ \pm}$; after unblinding, four resonances were found with significances greater than $5 \sigma$, which constitute the first direct observation of $\Sigma_{\mathrm{b}}^{(*)}$ baryons. The resonances are identified as the $\Sigma_{\mathrm{b}}^{ \pm}$and the $\Sigma_{\mathrm{b}}^{* \pm}$.

\subsubsection{Rare Decays}

In the field of rare decays, the large data sets of altogether more than 1 billion $B \bar{B}$ events obtained at the B factory experiments allow more and more precise measurements of $b \rightarrow s \gamma$ decay modes [56], which are interesting because in the Standard Model they are forbidden at tree level and proceed through loop diagrams. In extensions of the Standard Model, additional particles contribute to the loops, which may lead to observable deviations of the transition rates from the expected SM values. While the $b \rightarrow s \gamma$ decay channels are investigated with higher and higher precision, the large statistics now allows even the measurement of $b \rightarrow d \gamma$ transitions, which were first observed by Belle in 2005 [57]. The latest compilation from the Heavy Flavour Averaging Group [58] contains 20 measured $b \rightarrow s / d \gamma$ decay channels, with branching fractions as small as $4.6 \cdot 10^{-7}$ for $B^{0} \rightarrow \omega \gamma$ and precisions down to $6.5 \%$ for $B^{+} \rightarrow K^{+} \pi^{+} \pi^{-} \gamma$. The 2006 average for $b \rightarrow s \gamma$ decays [59] is now $B R\left(B \rightarrow X_{\mathrm{s}} \gamma\right)=(3.55 \pm 0.26) \cdot 10^{-4}$, which is on the high side of recent NNLO predictions $[56,60]$. The latest measurements from BaBar and Belle of $B \rightarrow \rho / \omega \gamma[61]$ are particularly impressive, measuring branching fractions around $10^{-6}$, in some cases with more than $5 \sigma$ significance. From a combination of the $b \rightarrow d / s \gamma$ measurements from Belle and BaBar, a constraint of $\left|V_{\mathrm{td}} / V_{\mathrm{ts}}\right|=0.202_{-0.016}^{+0.017}$ (exp.) \pm 0.015 (theor.) has been derived.

A very difficult, but interesting decay channel is $B^{+} \rightarrow \tau^{+} \bar{\nu}[56,62]$, which has been observed by Belle at $3.5 \sigma$ significance with a branching ratio of $B R\left(B^{+} \rightarrow \tau^{+} \bar{\nu}\right)=$ $\left(1.79_{-0.49}^{+0.56}{ }_{-0.46}^{+0.39}\right) \cdot 10^{-4}$, while BaBar measures $\left(0.88_{-0.67}^{+0.68} \pm 0.11\right) \cdot 10^{-4}$, or, translated into a $90 \%$ CL limit, $B R\left(B^{+} \rightarrow \tau^{+} \bar{\nu}\right)<1.8 \cdot 10^{-4}$. The combination of both results yields $(1.31 \pm 0.48) \cdot 10^{-4}$, corresponding to a $2.5 \sigma$ evidence. This result can be used to derive limits on the $H^{ \pm}$mass in SUSY models.

After the observation of $B_{\mathrm{s}}$ oscillations, the search for the decays $B_{\mathrm{s}, \mathrm{d}} \rightarrow \mu^{+} \mu^{-}$might be considered the next race in B physics. The Standard Model expectations for these decays are extremely small, and probably out of reach for current experiments: $B R\left(B_{\mathrm{s}} \rightarrow \mu^{+} \mu^{-}\right)=$ $(3.42 \pm 0.54) \cdot 10^{-9}$ and $B R\left(B_{\mathrm{d}} \rightarrow \mu^{+} \mu^{-}\right)=(1.0 \pm 0.14) \cdot 10^{-10}$. Again, these decays can only proceed through loop diagrams in the SM, and contributions from new particles may increase the rate by orders of magnitude [63]. Both Tevatron experiments have searched for the $B_{\mathrm{s}} \rightarrow \mu^{+} \mu^{-}$decay and have reported new, preliminary results based on new Run-II data. The limits are $B R\left(B_{\mathrm{s}} \rightarrow \mu^{+} \mu^{-}\right)<10 \cdot 10^{-8}\left(9.3 \cdot 10^{-8}\right)$ from CDF (D0); these limits correspond to branching ratios that are 29 (27) times larger than the SM expectation. CDF has also reported a preliminary limit for $B_{\mathrm{d}}$ decays: $B R\left(B_{\mathrm{d}} \rightarrow \mu^{+} \mu^{-}\right)<2.3 \cdot 10^{-8}$, which 
is 230 times greater than the SM expectation.

\subsection{Mixing and Oscillations}

While oscillations have long been established and thoroughly investigated in the $K^{0} \bar{K}^{0}$ and $B^{0} \bar{B}^{0}$ systems, until recently the frequency of the $B_{\mathrm{s}} \bar{B}_{\mathrm{s}}$ oscillations had not been measured, and no firm signal for mixing in the $D^{0} \bar{D}^{0}$ system had been observed. During the last year, both these gaps in our knowledge of neutral meson mixing have been filled.

The $B_{\mathrm{s}}$ oscillation frequency has now been measured by the Tevatron experiments [64]. After the first report on a double sided limit for the $B_{\mathrm{s}}$ oscillation frequency of $17<\Delta m_{\mathrm{s}}<$ $21 \mathrm{ps}^{-1}$ at $90 \% \mathrm{CL}$ from the D0 collaboration [65], CDF has for the first time observed a nonzero oscillation amplitude with more than $3 \sigma$ significance [66], at a frequency $\Delta m_{\mathrm{s}}=$ $17.77 \pm 0.10 \pm 0.07 \mathrm{ps}^{-1}$, consistent with the D0 result.

Meanwhile, the D0 collaboration has gone several steps further.

Based on a sample of $1.1 \mathrm{fb}^{-1}$ of decays $B_{\mathrm{s}} \rightarrow J / \psi \phi$, D0 has made a new measurement [67] of the difference between the lifetimes of the long and short lived $B_{\mathrm{s}}$ eigenstates. Both states can decay to the $J / \psi \phi$ final state, and by fitting the decay angle distributions their respective contributions to the sample at different eigentimes can be determined, with the result $\Delta \Gamma_{\mathrm{s}}=0.17 \pm 0.09 \pm 0.02 \mathrm{ps}^{-1}$. For the first time, the D0 collaboration has used this data also to extract the $\mathrm{CP}$ violating phase $\phi_{\mathrm{s}}$, which is the relative phase of the off-diagonal elements of the mass and decay matrices in the $B_{\mathrm{s}} \bar{B}_{\mathrm{s}}$ basis, from this data: $\phi_{\mathrm{s}}=-0.79 \pm 0.56$ (stat. $)_{-0.01}^{+0.14}$ (syst.). The SM prediction for $\phi_{\mathrm{s}}$ is very small, namely $\phi_{\mathrm{s}}=$ $(4.2 \pm 1.4) \cdot 10^{-3}[68]$.

D0 has also reported on a new measurement [69] of the branching ratio $B R\left(B_{\mathrm{s}} \rightarrow\right.$ $\left.D_{\mathrm{s}}^{(*)} D_{\mathrm{s}}^{(*)}\right)=0.039_{-0.017}^{+0.019}(\text { stat. })_{-0.017}^{+0.016}$ (syst.), which is lower than the only pre-existing measurement from ALEPH. This branching ratio is linked theoretically to the width difference $\Delta \Gamma_{\mathrm{s}}^{\mathrm{CP}}$ between the CP-even and odd $B_{\mathrm{s}}$ eigenstates. The resulting constraint is $\Delta \Gamma_{\mathrm{s}}^{\mathrm{CP}} / \Gamma_{\mathrm{s}}=0.079_{-0.035}^{+0.038}$ (stat.) ${ }_{-0.030}^{+0.031}$ (syst.).

While the difficulty in $B_{\mathrm{s}}$ mixing lies in the fact that the mixing is almost perfect because the oscillations occur much faster than the decay, the situation is reversed in the $D^{0} \bar{D}^{0}$ system. Here, the Standard Model prediction for the mixing parameters $x=\Delta m / \Gamma$ and $y=\Delta \Gamma / 2 \Gamma$ are very small [46], of the order $10^{-6 \ldots-2}$, which means that the $D^{0}$ decays much faster than one oscillation period lasts. Again, additional particles in the loop may increase the mixing, which could indicate new physics. In particular, new physics processes such as Flavour-Changing Neutral Currents, SUSY particles etc. would increase $x$ (i.e., the oscillation frequency), but not $y$ (the lifetime difference).

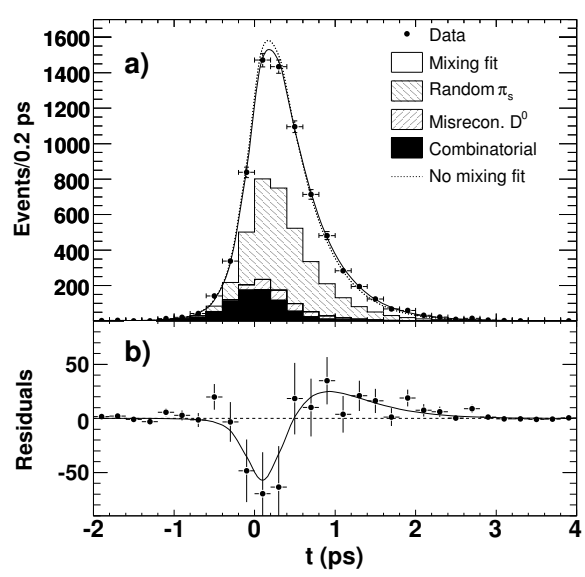

Figure 2: Proper time distribution of the $D^{0} \rightarrow K^{+} \pi^{-}$(wrong-sign WS) decays from BaBar

Until march of this year, the no-mixing case had been disfavoured with a significance of 
$2.1(2.3) \sigma$ by Belle (BaBar) analyses [70]. Then both collaborations published two papers titled "Evidence for $D^{0} \bar{D}^{0}$ mixing" side by side in Physics Review Letters [71], both reporting more than $3 \sigma$ evidence for $D^{0} \bar{D}^{0}$ oscillations.

The Belle analysis is based on $540 \mathrm{fb}^{-1}$ of data and measures the difference in apparent lifetime for the $\mathrm{CP}$ even decays $D^{0} \rightarrow K^{+} K^{-}, \pi^{+} \pi^{-}$to the lifetime of the decay $D^{0} \rightarrow$ $K^{-} \pi^{+}$. The data show indications for mixing with $3.2 \sigma$ significance. In addition, the Belle collaboration has performed a Dalitz analysis of the decay $D^{0} \rightarrow K_{\mathrm{S}}^{0} \pi^{-} \pi^{+}$, which provides the most sensitive result on $x$ to date: $x=(0.80 \pm 0.29 \pm 0.17) \%$ and disfavours the no-mixing solution with $2.7 \sigma$ significance [46, 72].

The BaBar analysis uses $384 \mathrm{fb}^{-1}$ of data and analyses the decays $D^{0} \rightarrow K^{-} \pi^{+}, K^{+} \pi^{-}$. The dominant decay mode is the right-sign (RS) decay mode to $K^{-} \pi^{+}$, while the wrongsign (WS) decay mode to $K^{+} \pi^{-}$may occur as doubly Cabibbo-suppressed (DCS) decay or through mixing. These two mechanisms are separated by the analysis of the time dependence of the decay, see Fig 2. From this data, BaBar can exclude the no-mixing hypothesis with $3.9 \sigma$.

\subsection{Future Experiments}

Looking ahead into the future, first beams at the LHC are now expected in early 2008. Heavy flavour physics will be of interest at the experiments ATLAS, CMS [73] and LHCb [74].

In ATLAS and CMS, the expected rates for beauty production are high: at a luminosity of $\mathcal{L}=10^{33} \mathrm{~cm}^{-2} \mathrm{~s}^{-1}$, around $10^{5} \mathrm{~b} \bar{b}$ pairs will be produced per second, which should allow high precision measurements despite the difficult environment. The big challenge here is to set up a reasonably efficient trigger, because bottom quarks are predominantly produced at relatively low transverse momenta, while the detectors are optimized for high- $p_{\mathrm{t}}$ discovery physics.

In the initial, "low" luminosity phase with expected luminosities around $\mathcal{L}=10^{33} \mathrm{~cm}^{-2} \mathrm{~s}^{-1}$, the ATLAS experiment envisages to use single particle triggers at Level 1, which will be refined by searches for more complex signatures in the High Level Trigger (HLT) [73]. Channels that have been studied include e.g. $B_{\mathrm{s}} \rightarrow D_{\mathrm{s}} \pi$ with subsequent decays $D_{\mathrm{s}} \rightarrow \phi \pi$, states involving a electromagnetic signature such as $J / \psi \rightarrow e e, K^{*} \gamma, \phi \gamma$, and final states with two muons. In the high luminosity mode at $\mathcal{L}=10^{34} \mathrm{~cm}^{-2} \mathrm{~s}^{-1}$, Level 1 will predominantly use the dimuon channel to identify $J / \psi$ or $B_{\mathrm{s}}$ decays to $\mu \mu$. CMS will also use muon triggers at Level 1, and plans to identify decay vertices from heavy flavour decays in the High Level trigger. A number of topics have been studied by both collaborations, such as the measurement of inclusive beauty cross sections (CMS), the measurement of $\sin 2 \beta$ in the golden mode $B^{0} \rightarrow J / \psi K_{\mathrm{S}}^{0}$ (ATLAS), analysis of $B_{\mathrm{s}} \rightarrow J / \psi \phi$ decays (ATLAS and CMS), and searches for rare decays such as $\Lambda_{\mathrm{b}} \rightarrow \Lambda \mu \mu, B^{0} \rightarrow K^{*} \mu \mu$ or $B_{\mathrm{s}} \rightarrow \phi \mu \mu$ (ATLAS). The ATLAS collaboration has also studied the prospects to measure $B_{\mathrm{s}}$ oscillations. They conclude that they could measure $\Delta m_{\mathrm{s}}$ with $5 \sigma$ significance at the current CDF value with the data from one year, i.e. $10 \mathrm{fb}^{-1}$.

CMS has also studied the prospects to measure the $B_{\text {c }}$ mass from decays to $J / \psi \pi$; the expected resolution from $1 \mathrm{fb}^{-1}$ of data is $22 \mathrm{MeV}$ statistical and $15 \mathrm{MeV}$ systematical uncertainty, which can be compared to the preliminary CDF result reported in this conference [55], based on $1.1 \mathrm{fb}^{-1}$, with statistical and systematic errors of 4.0 and $2.7 \mathrm{MeV}$, respectively. 
The LHCb experiment [74], is an experiment dedicated to the study of B physics at LHC. The detector is a single arm spectrometer, optimized to detect $\mathrm{b}$ hadrons at pseudo rapidities $1.9<\eta<4.9$, and will run at an interaction zone with a relatively low luminosity to reduce backgrounds, where one year of running at nominal luminosity will provide $2 \mathrm{fb}^{-1}$ of data. The LHCb collaboration has studied the prospects to measure the angles of the unitarity triangle; for $\sin 2 \beta$ they hope to achieve a statistical precision of 0.02 from one year of data taking in the golden channel $B^{0} \rightarrow J / \psi K_{\mathrm{S}}^{0}$. For the least well determined angle $\gamma$, the most promising method seems to be the analysis of decays $B^{0,+} \rightarrow D^{0} K^{0,+}$ with $D^{0}$ decays to $K \pi, K 3 \pi, \pi \pi$ and $K K$, with a comparison of the rates of Cabibbo Favoured and Doubly Cabibbo Suppressed decays. The estimated sensitivities are in the range $\sigma(\gamma) \approx 5^{\circ}-15^{\circ}$, depending on the actual value of $\gamma$.

An interesting benchmark is provided by the sensitivity of $B_{\mathrm{d}, \mathrm{s}} \rightarrow \mu \mu$, which has been studied by ATLAS, CMS [73], and LHCb [74]. Recall that currently CDF and D0 have reported upper limits of $100 \cdot 10^{-9}$ for the branching ratio [63] based on samples of $2 \mathrm{fb}^{-1}$ (D0) and $0.78 \mathrm{fb}^{-1}$ (CDF); these limits lie about 30 times above the SM expectation of $3.4 \cdot 10^{-9}$. A recent ATLAS study concludes that with an integrated luminosity of $30 \mathrm{fb}^{-1}$, corresponding to about three year's running in the low luminosity mode, an upper limit of $6.6 \cdot 10^{-9}$ at $90 \% \mathrm{CL}$ is achieveable, which is about a factor of two above the SM expectation. CMS concludes that it could provide a limit of $14 \cdot 10^{-9}$ (four times the SM expectation) with $10 \mathrm{fb}^{-1}$, i.e. one year's worth of data. $\mathrm{LHCb}$ on the other hand, which will operate at lower luminosities, claims a sensitivity that would allow a $3 \sigma$ observation of the decay $B_{\mathrm{s}} \rightarrow \mu \mu$ at the Standard Model branching ratio of $3.4 \cdot 10^{-9}$ with $2 \mathrm{fb}^{-1}$, corresponding to one year's worth of data [74] at nominal luminosity.

Looking even farther into the future, Tim Greenshaw reported on the applications of heavy flavour at the planned Innternational Linear Collider (ILC) [75], an $e^{+} e^{-}$collider with a centre-of-mass energy between 500 and $1000 \mathrm{GeV}$. The detector designs for this machine forsee a very good flavour separation power, which calls for vertex detectors with spectacular performance. Several groups are currently investigating various technologies that could fulfill the requirements for the inner pixel detector of an ILC detector. One important application of the heavy flavour identification capabilities of such a detector would be the measurement of the various branching fractions of a Higgs boson. Even if only one neutral Higgs boson were found at the LHC, this would allow to check wether these fractions are consistent with the predictions from the Standard Model, where only one Higgs doublet is assumed and therefore all branching fractions depend only on the mass of the single physical Higgs boson, or whether for instance up- and down-type fermions have different couplings to the Higgs, as expected for instance in Supersymmetric models, which have at least two Higgs doublets.

\subsection{Conclusions for the Experimental Part}

Heavy flavour production at HERA and Tevatron remains an interesting field of research, both from the experimental and the theory side. The experimental results become more and more precise, which makes it interesting to include them in the determination of parton densities, provided that the theoretical obstacles can be overcome and uncertainties arising from charm mass and fragmentation functions are treated consistently between experiment and theory. The beauty production data have taught us that the calculation of production cross sections do not always become more accurate for heavier quarks if there is sufficient 
phase space for QCD dynamics, which is something to keep in mind for LHC, where also the top will fall into this category.

During the last years we have seen a veritable renaissance of hadron spectroscopy. As the particle data book fills up, we see more and more results that indicate how incomplete our understanding of hadron structure still is, as illustrated by unexpected differences in hyperfine splittings in the charmonium sector [34], by unexpected states and masses in the $c \bar{s}$ system [39], and by the appearance of charmonium-like resonances for which we have no ready explanation $[34,39,46]$. While resonances that do not fit into the conventional $q \bar{q}$ picture have been known for a long time in the light meson sector, we now learn that also in the heavy quark sector there is more than is written in our philosophy. On the other hand, the investigation of rare decays such as $B^{+} \rightarrow \tau \nu$ or $B \rightarrow \mu \mu$ and other rare processes such as $D^{0} \bar{D}^{0}$ mixing, where we wait for results that would point to physics beyond the Standard Model, has once again failed to turn up anything unexpected.

\section{Theory}

In this section, we summarize the six theory contributions to the heavy-flavor working group, emphasizing computations that have been performed during the years 2006 and 2007 and have therefore not yet been presented at previous DIS workshops [76]. We start with new perturbative results for inclusive final states, i.e. heavy-quark structure functions and their relation to parton densities, and then move on to less inclusive final states, in particular quarkonium production and decay and heavy-quark spectroscopy. For the latter we emphasize new results from lattice QCD and QCD sum rules. Note that a series of new calculations on open heavy-quark production at various colliders in the general-mass variable-flavor number scheme (GM-VFNS) has been presented at this workshop in an introductory plenary talk by G. Kramer [77].

The heavy-quark coefficient functions for deep-inelastic lepton-hadron scattering (DIS) in the kinematic regime $Q^{2} \gg m^{2}$ have been calculated more than ten years ago in next-toleading order (NLO) of QCD by M. Buza et al., using operator product expansion techniques [78]. Here $Q^{2}$ and $m^{2}$ stand for the masses squared of the virtual photon and heavy quark respectively. The analytical results had been expressed in terms of 48 independent functions and had been used to check earlier, general calculations, which were, however, only accessible via large computer programs. J. Blümlein has now presented a re-calculation of the $\mathcal{O}\left(\alpha_{s}^{2}\right)$ massive operator matrix elements for the twist-2 operators, which contribute to the heavy flavor Wilson coefficients in unpolarized DIS in the region $Q^{2} \gg m^{2}$, using light-cone expansion techniques and confirming the above-cited calculation [79]. The application of the integration-by-parts method and harmonic sums in Mellin space allowed for a significant compactification of the results, which can now be expressed in terms of the basis $\left\{S_{1}, S_{2}, S_{3}, S_{-2}, S_{-3}\right\}$ and $S_{-2,1}$, i.e. of only two independent functions.

While the proton is just a simple $|u u d\rangle$ Fock state in the quark model, the possibility of an intrinsic-charm, i.e. a $|u u d c \bar{c}\rangle$, component has repeatedly been put forward in the context of light-cone [80] or meson-cloud models [81, 82]. W.K. Tung et al. have performed global fits of parton density functions (PDFs), assuming that the charm-density is not only generated radiatively at $\mu=m_{c}$ and then evolved to $Q$, but allowing for the possibility of light-cone, meson-cloud, or sea-quark like intrinsic charm density [83]. The quality of each fit is measured by a global $\chi^{2}$, shown in Fig. 3 as a function of the momentum fraction $\langle x\rangle_{c+\bar{c}}$ carried by the charm quark at the starting scale $\mu=m_{c}=1.3 \mathrm{GeV}$. For $\langle x\rangle_{c+\bar{c}} \leq$ 
0.01, the quality of the fit varies very little, i.e. the global analysis of hard-scattering data provides no evidence either for or against intrinsic charm. Above this point, all three curves in Fig. 3 rise steeply with $\langle x\rangle_{c+\bar{c}}$, so that global fits do place useful upper bounds on intrinsic charm. There is no data set that is particularly sensitive to intrinsic charm, but the global QCD analysis rules out the possibility of an intrinsic charm component much larger than 0.02 in momentum fraction. A variation of the charm quark mass $m_{c}$ shows that the data prefer lower masses around $1.3 \mathrm{GeV}$ with respect to higher masses of $1.5 \mathrm{GeV}$. The difference in $\chi^{2}$ is in this case almost entirely due to the charm contribution to the proton structure function $F^{2}$, which has been precisely measured at HERA.

Turning to less inclusive quantities, the production and decay of heavy quarkantiquark bound states (quarkonia $\mathcal{Q}$ ) is still far from understood. While the effective field theory of non-relativistic QCD

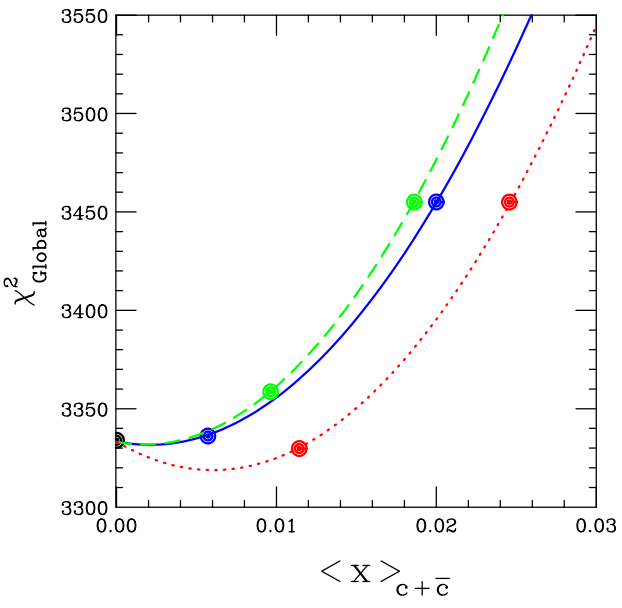

Figure 3: Goodness-of-fit vs. momentum fraction of intrinsic charm at the starting scale $\mu=1.3 \mathrm{GeV}$ for the light-cone (solid curve), meson-cloud (dashed curve), and sealike model (dotted curve). (NRQCD) has long been believed to be phenomenologically successful and is still believed to be theoretically more consistent than the color-singlet model (CSM), recent Tevatron Run II data on the polarization of large- $p_{T} J / \Psi$ and $\Upsilon$-mesons do not support the idea that their production is dominated by color-octet fragmentation processes as predicted in NRQCD. Since higher-order QCD corrections are largely unknown (except for color-singlet photoproduction [84], color-singlet and color-octet production in photon-photon collisions $[85,86]$ and a very recent calculation for direct colorsinglet hadroproduction [87]), other theoretical frameworks continue to be investigated. One example is the $k_{T}$-factorization formalism, where the production cross section

$$
\begin{aligned}
\mathrm{d} \sigma(p \bar{p} \rightarrow \mathcal{Q} & +X)=\int \frac{\mathrm{d} x_{1}}{x_{1}} \int \mathrm{d} k_{1 T}^{2} \int \frac{\mathrm{d} \phi_{1}}{2 \pi} \Phi\left(x_{1}, k_{1 T}^{2}, \mu^{2}\right) \\
& \times \int \frac{\mathrm{d} x_{2}}{x_{2}} \int \mathrm{d} k_{2 T}^{2} \int \frac{\mathrm{d} \phi_{2}}{2 \pi} \Phi\left(x_{2}, k_{2 T}^{2}, \mu^{2}\right) \mathrm{d} \hat{\sigma}(R R \rightarrow \mathcal{Q}+X)
\end{aligned}
$$

is computed by a double convolution of unintegrated parton densities $\Phi$ and partonic cross section $\hat{\sigma}$ over longitudinal momentum fractions $x_{1,2}$ and intrinsic transverse momenta $k_{1 T, 2 T}$ of the reggeized partons $R$ in the (anti-)proton $p(\bar{p})$. This method has been applied by Kniehl et al. to charmonium production at different colliders [88] and now, as V.A. Saleev reported, also to bottomonium hadroproduction at the Tevatron [89]. The long-distance operator matrix elements (OMEs) of NRQCD have been refitted to the $p_{T}$-spectra of prompt $\Upsilon$ mesons at the Tevatron. Since the intrinsic $k_{T}$ leads to a harder $p_{T}$-spectrum already for the color-singlet contributions, the color-octet OMEs turn out to be considerably smaller and in many cases even consistent with zero, as their values are not sufficiently constrained by the data. In addition, the results depend strongly on the assumed unintegrated parton 
densities, which are poorly known and only constrained to agree with the integrated ones,

$$
x f\left(x_{1,2}, \mu^{2}\right)=\int_{0}^{\mu^{2}} \mathrm{~d} k_{1 T, 2 T}^{2} \Phi\left(x_{1,2}, k_{1 T, 2 T}^{2}, \mu^{2}\right) .
$$

As can be seen from Tab. 1, the quality of the fit, measured by $\chi^{2}$ per degree of freedom,

Table 1: OMEs of the $\Upsilon(1 S, 2 S, 3 S)$ and $\chi_{b 0}(1 P, 2 P)$ mesons from fits to CDF data from Runs I and II in the Regge-kinematics approach using unintegrated gluon distributions by J. Blümlein (JB), Jung and Salam (JS), and Kimber, Martin and Ryskin (KMR).

\begin{tabular}{|c|c|c|c|}
\hline NME & Fit JB & Fit JS & Fit KMR \\
\hline$\left.\left\langle\mathcal{O}^{\Upsilon(1 S)}{ }^{3} S_{1}^{(1)}\right]\right\rangle / \mathrm{GeV}^{3}$ & $10.9 \pm 1.6$ & $10.9 \pm 1.6$ & $10.9 \pm 1.6$ \\
\hline$\left\langle\mathcal{O}^{\Upsilon(1 S)}\left[{ }^{3} S_{1}^{(8)}\right]\right\rangle / \mathrm{GeV}^{3}$ & $(5.3 \pm 0.5) \times 10^{-3}$ & $(0.0 \pm 1.8) \times 10^{-4}$ & $(0.0 \pm 3.1) \times 10^{-3}$ \\
\hline$\left\langle\mathcal{O}^{\Upsilon(1 S)}\left[{ }^{1} S_{0}^{(8)}\right]\right\rangle / \mathrm{GeV}^{3}$ & $(0.0 \pm 4.7) \times 10^{-4}$ & $(0.0 \pm 5.2) \times 10^{-5}$ & $(0.0 \pm 4.3) \times 10^{-3}$ \\
\hline$\left\langle\mathcal{O}^{\Upsilon(1 S)}\left[{ }^{3} P_{0}^{(8)}\right]\right\rangle / \mathrm{GeV}^{5}$ & $(0.0 \pm 1.3) \times 10^{-3}$ & $(0.0 \pm 1.6) \times 10^{-4}$ & $(9.5 \pm 2.0) \times 10^{-2}$ \\
\hline$M_{5}^{\Upsilon(1 S)} / \mathrm{GeV}^{3}$ & $(0.0 \pm 7.6) \times 10^{-4}$ & $(0.0 \pm 8.7) \times 10^{-5}$ & $(2.1 \pm 0.9) \times 10^{-2}$ \\
\hline$\left\langle\mathcal{O}^{\chi} \chi_{b 0}(1 P)\left[{ }^{3} P_{0}^{(1)}\right]\right\rangle / \mathrm{GeV}^{5}$ & $2.4 \pm 0.4$ & $2.4 \pm 0.4$ & $2.4 \pm 0.4$ \\
\hline$\left\langle\mathcal{O}^{\chi_{b 0}(1 P)}\left[{ }^{3} S_{1}^{(8)}\right]\right\rangle / \mathrm{GeV}^{3}$ & $(0.0 \pm 2.1) \times 10^{-3}$ & $(0.0 \pm 8.4) \times 10^{-5}$ & $(0.0 \pm 1.4) \times 10^{-3}$ \\
\hline$\left\langle\mathcal{O}^{\Upsilon(2 S)}\left[{ }^{3} S_{1}^{(1)}\right]\right\rangle / \mathrm{GeV}^{3}$ & $4.5 \pm 0.7$ & $4.5 \pm 0.7$ & $4.5 \pm 0.7$ \\
\hline$\left\langle\mathcal{O}^{\Upsilon(2 S)}\left[{ }^{3} S_{1}^{(8)}\right]\right\rangle / \mathrm{GeV}^{3}$ & $(0.0 \pm 5.9) \times 10^{-3}$ & $(0.0 \pm 4.1) \times 10^{-4}$ & $(3.3 \pm 0.8) \times 10^{-2}$ \\
\hline$\left\langle\mathcal{O}^{\Upsilon(2 S)}\left[{ }^{1} S_{0}^{(8)}\right]\right\rangle / \mathrm{GeV}^{3}$ & $(0.0 \pm 9.2) \times 10^{-4}$ & $(0.0 \pm 8.3) \times 10^{-5}$ & $(0.0 \pm 3.7) \times 10^{-3}$ \\
\hline$\left\langle\mathcal{O}^{\Upsilon(2 S)}\left[{ }^{3} P_{0}^{(8)}\right]\right\rangle / \mathrm{GeV}^{5}$ & $(0.0 \pm 2.6) \times 10^{-3}$ & $(0.0 \pm 2.8) \times 10^{-4}$ & $(0.0 \pm 1.6) \times 10^{-2}$ \\
\hline$M_{5}^{\Upsilon(2 S)} / \mathrm{GeV}^{3}$ & $(0.0 \pm 1.5) \times 10^{-3}$ & $(0.0 \pm 1.4) \times 10^{-4}$ & $(0.0 \pm 7.2) \times 10^{-3}$ \\
\hline$\left\langle\mathcal{O}^{\chi}{ }_{b 0}(2 P)\left[{ }^{3} P_{0}^{(1)}\right]\right\rangle / \mathrm{GeV}^{5}$ & $2.6 \pm 0.5$ & $2.6 \pm 0.5$ & $2.6 \pm 0.5$ \\
\hline$\left\langle\mathcal{O}^{\chi} \chi_{b 0}(2 P)\left[{ }^{3} S_{1}^{(8)}\right]\right\rangle / \mathrm{GeV}^{3}$ & $(1.1 \pm 0.4) \times 10^{-2}$ & $(0.0 \pm 2.8) \times 10^{-4}$ & $(0.0 \pm 5.7) \times 10^{-3}$ \\
\hline$\left\langle\mathcal{O}^{\Upsilon(3 S)}\left[{ }^{3} S_{1}^{(1)}\right]\right\rangle / \mathrm{GeV}^{3}$ & $4.3 \pm 0.9$ & $4.3 \pm 0.9$ & $4.3 \pm 0.9$ \\
\hline$\left\langle\mathcal{O}^{\Upsilon(3 S)}\left[{ }^{3} S_{1}^{(8)}\right]\right\rangle / \mathrm{GeV}^{3}$ & $(1.4 \pm 0.3) \times 10^{-2}$ & $(5.9 \pm 4.2) \times 10^{-3}$ & $(1.1 \pm 0.4) \times 10^{-2}$ \\
\hline$\left\langle\mathcal{O}^{\Upsilon(3 S)}\left[{ }^{1} S_{0}^{(8)}\right]\right\rangle / \mathrm{GeV}^{3}$ & $(0.0 \pm 2.6) \times 10^{-3}$ & $(0.0 \pm 8.1) \times 10^{-4}$ & $(0.0 \pm 2.7) \times 10^{-3}$ \\
\hline$\left\langle\mathcal{O}^{\Upsilon(3 S)}\left[{ }^{3} P_{0}^{(8)}\right]\right\rangle / \mathrm{GeV}^{5}$ & $(2.4 \pm 0.8) \times 10^{-2}$ & $(3.4 \pm 4.2) \times 10^{-3}$ & $(5.2 \pm 1.1) \times 10^{-2}$ \\
\hline$M_{5}^{\Upsilon(3 S)} / \mathrm{GeV}^{3}$ & $(5.2 \pm 4.4) \times 10^{-3}$ & $(7.4 \pm 10.2) \times 10^{-4}$ & $(1.1 \pm 0.5) \times 10^{-2}$ \\
\hline $\begin{array}{c}\left\langle\mathcal{O}^{\chi_{b 0}(3 P)}\left[{ }^{3} P_{0}^{(1)}\right]\right\rangle / \mathrm{GeV}^{5} \\
\chi^{2} / \text { d.o.f. }\end{array}$ & $\begin{array}{l}2.7 \pm 0.7 \\
2.9\end{array}$ & $\begin{array}{c}2.7 \pm 0.7 \\
27\end{array}$ & $\begin{array}{l}2.7 \pm 0.7 \\
0.5\end{array}$ \\
\hline
\end{tabular}

varies widely with the PDFs from 0.5 to 27 .

Quarkonium decay OMEs can be computed from first principles using lattice QCD, so that their determination becomes independent of fits to experimental data or potential model assumptions. A. Hart reported about a recent result that matches the electromagnetic vector-annihilation current in lattice NRQCD to the one in continuum QCD, which should allow for a prediction of the leptonic decay widths of $S$-wave bottomonia with ten percent accuracy and of the ratio of the $2 \mathrm{~S}$ and $1 \mathrm{~S} \Upsilon$-states with one percent accuracy [90]. Numerical results should be available soon.

Charmonium $\left(\eta_{c}\right.$ and $\left.\chi_{c 0}\right)$ decays into two photons have been calculated for the first time by Dudek and Edwards, albeit only in the quenched approximation, using relativistic 
valence quarks and a perturbative expansion of the photon-quark coupling, which allows to replace the photon by a superposition of QCD states [91]. While the width obtained for the $\chi_{c 0}(2.41 \pm 0.58 \pm 0.72 \pm 0.48 \mathrm{keV})$ is in rather good agreement with the experimental value $(2.84 \pm 0.40 \mathrm{keV})$, the one for the $\eta_{c}(2.65 \pm 0.26 \pm 0.80 \pm 0.53 \mathrm{keV})$ is smaller by a factor of three $(7.14 \pm 2.49 \mathrm{keV})$. This might be due to an incorrect running of the strong coupling constant and a depleted wave function at the origin in this calculation. In addition, the discretization error might not be reliable, as only one lattice spacing has been used.

Lansberg and Pham have computed the two-photon widths of ground-state and radially excited $\eta_{c}[92]$ and $\eta_{b}$ mesons [93] with an effective Lagrangian in the static approximation, taking into account binding energy effects for the radial excitations. Using heavy-quark spin symmetry, they assume equality of the $f_{\eta_{c}}\left(f_{\eta_{c^{\prime}}}\right)$ and $f_{J / \Psi}\left(f_{\Psi^{\prime}}\right)$ decay constants and relate the two-photon width of the $\eta_{c}$ to the leptonic decay width of the $J / \Psi$. While good agreement with the experimental value is found for the $\eta_{c}$ ground state $(7.5-10 \mathrm{keV})$, their result for $\eta_{c}^{\prime}(3.5-4.5 \mathrm{keV})$ is three times larger than the CLEO measurement $(1.3 \pm 0.6 \mathrm{keV})$. This may be due to the fact that $f_{\eta_{c}^{\prime}}$ is not equal, but three times smaller than $f_{\Psi^{\prime}}$, according to a recent lattice calculation in the quenched approximation by Dudek and Edwards [94].

Radiative decays of charmonia to light mesons have recently been computed in perturbative QCD [95], albeit keeping only the color-singlet wave function contribution and assuming the light mesons to be also described by non-relativistic color-singlet $q \bar{q}$ bound states with finite constituent quark masses. These have then been used to regularize the one-loop diagrams. The numerical results are given in Tab. 2. Unfortunately a systematic study on the theoretical uncertainties with the chosen masses and scales and with respect to possible

Table 2: Proposed theoretical and measured experimental values for $\mathcal{B}\left(J / \psi \rightarrow f_{J} \gamma\right)$.

\begin{tabular}{|c|ccccc|}
\hline & $f_{0}(980)$ & $f_{1}(1285)$ & $f_{2}(1270)$ & $f_{1}^{\prime}(1420)$ & $f_{2}^{\prime}(1525)$ \\
\hline $\mathcal{B}_{t h} \times 10^{4}$ & 1.6 & 7.0 & 8.7 & 1.8 & 2.0 \\
$\mathcal{B}_{e x} \times 10^{4}$ & - & $6.1 \pm 0.8$ & $13.8 \pm 1.4$ & $7.9 \pm 1.3$ & $4.5_{-0.4}^{+0.7}$ \\
\hline
\end{tabular}

color-octet contributions has not been performed. The calculated $J / \psi$-decay branching ratios to the $P$-wave mesons $f_{2}(1270)$ and $f_{1}(1285)$ fit the data well, while that of $f_{0}(980)$ (if treated as an $s \bar{s}$ meson) is predicted to be $1.6 \times 10^{-4}$, which implies that $f_{0}(1710)$ can not be the $s \bar{s}$ or $(u \bar{u}+d \bar{d}) / \sqrt{2}$ meson. Decays of $P$-wave charmonia $\chi_{c J} \rightarrow \rho(\omega, \phi) \gamma(J=0,1,2)$ have also been studied. The branching ratio of $\chi_{c 1} \rightarrow \rho \gamma$ is predicted to be $1.4 \times 10^{-5}$, which may be tested by CLEO-c and BESIII with future experiments.

A similar calculation has been performed for the radiative decays of bottomonia into charmonia and light mesons, taking into account in addition the sometimes significant QED contributions [96]. While the results for radiative decays into truly non-relativistic charmonia are likely to be more reliable than those for radiative decays into (in reality relativistic) light mesons, these decays remain to be observed. On the other hand, the calculated branching ratios for $\Upsilon \rightarrow f_{2}(1270) \gamma\left(6.3 \times 10^{-5}\right)$ and $\Upsilon \rightarrow f_{2}^{\prime}(1525) \gamma\left(2.0 \times 10^{-5}\right)$ are only barely consistent with recent CLEO data $\left[(1.0 \pm 0.1) \times 10^{-4}\right.$ and $\left.(3.7 \pm 1.2) \times 10^{-5}\right]$, suggesting that the theoretical approach may still need improvement.

Finally, J.M. Richard reviewed the current status of light and heavy multiquark spectroscopy [97]. While experimental evidence for the $u u d d \bar{s}$ pentaquark $\theta^{+}$seems to vanish, a single-charm baryon with a mass of $2940 \mathrm{MeV}$ and a width of approximately $17 \mathrm{MeV}$ 
has recently been confirmed by BaBar, and a double-charm baryon with a mass of 3520 $\mathrm{MeV}$ may have been observed by the Selex collaboration. Potential models have since long predicted the existence of baryons with at least one heavy quark, assuming them to be diquark-quark systems that can be described with hyperspherical coordinates. In the meson sector, the state $X(3940)$ may be a $1^{-+}$candidate for a $c c g$ hybrid state, to be described in the Born-Oppenheimer approximation with classical constituent gluons. A second recently confirmed state is the $X(3872)$, which QCD sum rules predict to be a $1^{++} c \bar{c} q \bar{q}$ bound state, even if the theoretical mass found is slightly larger $(3925 \pm 127 \mathrm{MeV})$ [98].

\section{Acknowledgments}

We thank all the speakers of the heavy-flavor working group for their contributions and the local committee for the perfect organization of the DIS 2007 workshop.

\section{References}

[1] B. List, "Heavy flavour WG summary - Experiment," slides available at http://indico.cern. ch/contributionDisplay.py? contribId=19\&sessionId=2\&conf Id=9499

[2] H. Stadie [ZEUS Collab.], "DIS charm cross sections through $D^{*}$ and $D$ meson tagging by the ZEUS detector," these proceedings.

[3] D. Nicholass [ZEUS Collab.], " $D^{ \pm}$cross sections in DIS using the ZEUS Micro Vertex Detector," these proceedings.

[4] K. Lipka [H1 Collab.], "Charm production in DIS at H1," these proceedings.

[5] S. Chekanov et al. [ZEUS Collab.], Phys. Lett. B 649, 111 (2007).

[6] B. W. Harris and J. Smith, Phys. Rev. D 57, 2806 (1998).

[7] S. Chekanov et al. [ZEUS Collab.], arXiv:0704.3562 [hep-ex].

[8] S. Fang [ZEUS Collab.], "Charm fragmentation function and fragmentation functions of charm mesons at ZEUS," these proceedings.

[9] S. Chekanov et al. [ZEUS Collab.], Eur. Phys. J. C 44, 351 (2005).

[10] S. Schmidt [H1 Collab.], "Charm production with jets at H1," these proceedings.

[11] A. Aktas et al. [H1 Collab.], arXiv:hep-ex/0701023.

[12] H. Jung, Comput. Phys. Commun. 143 (2002) 100.

[13] A. Aktas et al. [H1 Collab.], Eur. Phys. J. C 50, 251 (2007).

[14] A. Geiser, "Review of beauty production at HERA," these proceedings.

[15] B. Reisert [CDF and D0 Collab.], "Charm and beauty production at the Tevatron," these proceedings.

[16] B. Kahle [ZEUS Collab.], " $F_{2}^{b b}$ from the ZEUS HERA-II data," these proceedings.

[17] A. Aktas et al. [H1 Collaboration], Eur. Phys. J. C 40 (2005) 349,

A. Aktas et al. [H1 Collaboration], Eur. Phys. J. C 45 (2006) 23.

[18] K. Krüger, "Review on charmonium production," these proceedings.

[19] E. L. Berger and D. L. Jones, Phys. Rev. D 23, 1521 (1981), R. Baier and R. Rückl, Phys. Lett. B 102, 364 (1981).

[20] F. Abe et al. [CDF Collab.], Phys. Rev. Lett. 79, 572 (1997), T. Shears [CDF Collab.], Eur. Phys. J. C 33, S475 (2004).

[21] G. T. Bodwin, E. Braaten and G. P. Lepage, Phys. Rev. D 51, 1125 (1995) [Erratum-ibid. D 55, 5853 (1997)].

[22] A. Abulencia et al. [CDF Collab.], arXiv:hep-ex/0703028.

[23] M. Steder [H1 Collab.], "Inelastic $J / \psi$ production in DIS at H1," these proceedings.

[24] M. Krämer, Nucl. Phys. B 459, 3 (1996).

[25] C. Adloff et al. [H1 Collab.], Eur. Phys. J. C 25, 25 (2002),

S. Chekanov et al. [ZEUS Collab.], Eur. Phys. J. C 27, 173 (2003). 
[26] M. zur Nedden [HERA-B Collab.], "Recent charmonium results from HERA-B," these proceedings.

[27] W. A. Zajc, "The fluid nature of quark-gluon-plasma," these proceedings.

[28] D. Hornback [PHENIX Collab.], "Measurements of heavy quark production via single leptons in $p+p$ and $A u+A u$ collisions at $\sqrt{s}=200 \mathrm{GeV}$," these proceedings.

[29] A. Mischke [STAR Collab.], "Recent heavy flavour results from STAR," these proceedings.

[30] A. Adare et al. [PHENIX Collab.], Phys. Rev. Lett. 98, 172301 (2007).

[31] B. I. Abelev et al. [STAR Collab.], arXiv:nucl-ex/0607012.

[32] E. T. Atomssa [PHENIX Collab.], " $J / \psi$ suppression measurements by the PHENIX experiment at RHIC," these proceedings.

[33] A. Adare et al. [PHENIX Collab.], arXiv:nucl-ex/0611020.

[34] K. K. Seth [CLEO-c Collab.], "Charm at CLEO-c," these proceedings.

[35] S. K. Choi et al. [Belle Collab.], Phys. Rev. Lett. 89, 102001 (2002) [Erratum-ibid. 89, 129901 (2002)], D. M. Asner et al. [CLEO Collab.], Phys. Rev. Lett. 92, 142001 (2004), K. Abe et al. [Belle Collab.], arXiv:hep-ex/0507019.

[36] J. L. Rosner et al. [CLEO Collab.], Phys. Rev. Lett. 95, 102003 (2005).

[37] W. M. Yao et al. [Particle Data Group], J. Phys. G 33, 1 (2006).

[38] C. Cawlfield et al. [CLEO Collab.], Phys. Rev. Lett. 98, 092002 (2007).

[39] V. Poireau [Belle and BaBar Collab.], "New resonances and meson spectroscopy at Belle and BaBar," these proceedings.

[40] G. Gokhroo et al. [Belle Collab.], Phys. Rev. Lett. 97, 162002 (2006).

[41] B. Aubert et al. [BaBar Collab.], arXiv:hep-ex/0607083.

[42] T. E. Coan et al. [CLEO Collab.], Phys. Rev. Lett. 96, 162003 (2006).

[43] Q. He et al. [CLEO Collab.], Phys. Rev. D 74, 091104 (2006).

[44] B. Aubert et al. [BaBar Collab.], Phys. Rev. Lett. 95, 142001 (2005).

[45] K. Abe et al. [Belle Collab.], arXiv:hep-ex/0612006.

[46] G. Pakhlova [Belle and BaBar Collab.], "Charm physics at B factories," these proceedings.

[47] K. Abe et al. [Belle Collab.], Phys. Rev. Lett. 98, 092001 (2007).

[48] H. Mendez [CLEO Collab.], arXiv:hep-ex/0702008.

[49] B. Aubert et al. [BaBar Collab.], arXiv:hep-ex/0610057.

[50] K. Abe et al. [Belle Collab.], arXiv:hep-ex/0507019,

K. Abe et al. [Belle Collab.], Phys. Rev. Lett. 94, 182002 (2005),

S. Uehara et al. [Belle Collab.], Phys. Rev. Lett. 96, 082003 (2006).

[51] B. Aubert et al. [BaBar Collab.], Phys. Rev. Lett. 97, 222001 (2006).

[52] B. Aubert et al. [BaBar Collab.], Phys. Rev. Lett. 98, 012001 (2007), K. Abe et al. [Belle Collab.], arXiv:hep-ex/0608043.

[53] R. Chistov et al. [Belle Collab.], Phys. Rev. Lett. 97, 162001 (2006), B. Aubert et al. [BaBar Collab.], arXiv:hep-ex/0607042.

[54] B. Aubert et al. [BaBar Collab.], Phys. Rev. Lett. 97, 232001 (2006).

[55] M. Heck [CDF and D0 Collab.], "Recent results on B spectroscopy at the Tevatron," these proceedings.

[56] I. G. Eschrich [BaBar Collab.], "Rare decays at the B Factories," these proceedings.

[57] K. Abe et al. [Belle Collab.], Phys. Rev. Lett. 96, 221601 (2006).

[58] E. Barberio et al. [Heavy Flavor Averaging Group (HFAG)], arXiv:0704.3575 [hep-ex].

[59] E. Barberio et al. [Heavy Flavor Averaging Group (HFAG)], arXiv:hep-ex/0603003.

[60] M. Misiak et al., Phys. Rev. Lett. 98, 022002 (2007), T. Becher and M. Neubert, Phys. Rev. Lett. 98, 022003 (2007).

[61] K. Abe et al. [Belle Collab.], Phys. Rev. Lett. 96, 221601 (2006), B. Aubert et al. [BaBar Collab.], Phys. Rev. Lett. 98, 151802 (2007).

[62] K. Ikado et al. [Belle Collab.], Phys. Rev. Lett. 97, 251802 (2006),

B. Aubert et al. [BaBar Collab.], arXiv:hep-ex/0608019.

[63] M. Corcoran [CDF and D0 Collab.], "B hadron rare decays and lifetimes," these proceedings.

[64] R. Kehoe [CDF and D0 Collab.], " $B_{\mathrm{s}}$ mixing and lifetime difference measurements," these proceedings. 
[65] V. M. Abazov et al. [D0 Collab.], Phys. Rev. Lett. 97, 021802 (2006).

[66] A. Abulencia et al. [CDF Collab.], Phys. Rev. Lett. 97, 062003 (2006).

[67] V. M. Abazov et al. [D0 Collab.], Phys. Rev. Lett. 98, 121801 (2007).

[68] A. Lenz and U. Nierste, arXiv:hep-ph/0612167.

[69] V. M. Abazov et al. [D0 Collab.], arXiv:hep-ex/0702049.

[70] L. M. Zhang et al. [Belle Collab.], Phys. Rev. Lett. 96, 151801 (2006), B. Aubert et al. [BaBar Collab.], Phys. Rev. Lett. 97, 221803 (2006).

[71] B. Aubert et al. [BaBar Collab.], Phys. Rev. Lett. 98, 211802 (2007), M. Staric et al. [Belle Collab.], Phys. Rev. Lett. 98, 211803 (2007).

[72] K. Abe et al. [Belle Collab.], arXiv:0704.1000 [hep-ex].

[73] A. Krasznahorkay Jr. [ATLAS and CMS Collab.], "Outlook for b and c physics at the LHC in ATLAS and CMS," these proceedings.

[74] E. Santovetti [LHCb Collab.], "B physics prospects at LHCb," these proceedings.

[75] T. Greenshaw, "Physics with flavour at the International Linear Collider," these proceedings.

[76] M. Klasen, "Heavy Flavour WG - Theory summary," slides available at http: //indico . cern. ch/contributionDisplay . py? contribId=19\&sessionId=2\&conf Id=9499

[77] G. Kramer, "Recent developments in heavy flavour production," these proceedings.

[78] M. Buza, Y. Matiounine, J. Smith, R. Migneron and W. L. van Neerven, Nucl. Phys. B 472, 611 (1996).

[79] I. Bierenbaum, J. Blümlein and S. Klein, arXiv:hep-ph/0703285 and these proceedings.

[80] S. J. Brodsky, P. Hoyer, C. Peterson and N. Sakai, Phys. Lett. B 93, 451 (1980).

[81] F. S. Navarra, M. Nielsen, C. A. A. Nunes and M. Teixeira, Phys. Rev. D 54, 842 (1996).

[82] W. Melnitchouk and A. W. Thomas, Phys. Lett. B 414, 134 (1997).

[83] J. Pumplin, H. L. Lai and W. K. Tung, Phys. Rev. D 75, 054029 (2007) and these proceedings.

[84] M. Krämer, J. Zunft, J. Steegborn and P. M. Zerwas, Phys. Lett. B 348, 657 (1995).

[85] M. Klasen, B. A. Kniehl, L. N. Mihaila and M. Steinhauser, Nucl. Phys. B 713, 487 (2005).

[86] M. Klasen, B. A. Kniehl, L. N. Mihaila and M. Steinhauser, Phys. Rev. D 71, 014016 (2005).

[87] J. Campbell, F. Maltoni and F. Tramontano, arXiv:hep-ph/0703113.

[88] B. A. Kniehl, D. V. Vasin and V. A. Saleev, Phys. Rev. D 73, 074022 (2006).

[89] B. A. Kniehl, V. A. Saleev and D. V. Vasin, Phys. Rev. D 74, 014024 (2006) and these proceedings.

[90] A. Hart, G. M. von Hippel and R. R. Horgan, Phys. Rev. D 75, 014008 (2007) and these proceedings.

[91] J. J. Dudek and R. G. Edwards, Phys. Rev. Lett. 97, 172001 (2006).

[92] J. P. Lansberg and T. N. Pham, Phys. Rev. D 74, 034001 (2006)

[93] J. P. Lansberg and T. N. Pham, Phys. Rev. D 75, 017501 (2007).

[94] J. J. Dudek, R. G. Edwards and D. G. Richards, Phys. Rev. D 73, 074507 (2006).

[95] Y. J. Gao, Y. J. Zhang and K. T. Chao, Chin. Phys. Lett. 23, 2376 (2006).

[96] Y. J. Gao, Y. J. Zhang and K. T. Chao, arXiv:hep-ph/0701009.

[97] J. M. Richard, Nucl. Phys. Proc. Suppl. 164, 131 (2007) and these proceedings.

[98] R. D. Matheus, S. Narison, M. Nielsen and J. M. Richard, Phys. Rev. D 75, 014005 (2007). 